\title{
MC-CDMA Transmission with Blanking Nonlinearity for Impulsive Noise Power-Line Communication Channels
}

\author{
Khaled M. Rabie and Emad Alsusa \\ Microwave and Communication Systems (MACS) Group, \\ School of Electrical and Electronic Engineering, \\ The University of Manchester, Manchester, UK, M13 9PL, \\ Email: \{khaled.rabie, e.alsusa\}@ manchester.ac.uk
}

\begin{abstract}
In this paper we investigate the application of multicarrier code division multiple access (MC-CDMA) in impulsive noise power-line channels. In order to improve system performance, impulsive noise blanking is utilized at the receiver's front-end. For comparison-sake, three families of spreading codes are considered here, namely, pseudonoise (PN), Walsh-Hadamard (WH) and poly-phase (PP) sequences. Probability of blanking error $\left(P_{b}\right)$ and output signal-to-noise ratio $(\mathbf{S N R})$ performances are adopted to characterize the achievable gains. The results reveal that the proposed scheme is able to achieve considerable improvements in terms of $P_{b}$ and output SNR performance. It is also shown that PP-based MC-CDMA yields the best overall performance with a 1.2 dB SNR gain relative to blanking-based orthogonal frequency division multiple access systems.
\end{abstract}

Index Terms-Blanking, impulsive noise, MC-CDMA, polyphase sequences, power-line communications (PLC), pseudonoise codes, symbol error rate (SER), Walsh-Hadamard sequences.

\section{INTRODUCTION}

Power-line communication (PLC) technology exploits the existing electrical power supply networks for data transmission. Unlike many other communication channels, powerlines (PLs) are particularly sensitive to high frequency-dependent attenuation, fluctuating impedance and non-Gaussian interference [1]. In such environments, multi-carrier transmission such as orthogonal frequency-division multiplexing (OFDM) and codedivision multiple access (CDMA) seem to be the most appropriate interfaces due to their robustness against frequencyselectivity and interference in comparison to single-carrier techniques [1]-[4]. As such OFDM has been adopted by many existing PLC standards [5].

It is widely known that noise over PLs remains the most detrimental element influencing communication signals and is, generally, classified into two categories: background noise and impulsive noise, with the latter being the most dominant one which is characterized by high amplitude, short duration, and random probability of occurrence. Numerous methods have been introduced in the literature to diminish the adverse effect of impulsive noise; the most common of which is the application of a nonlinear preprocessor, such as blanking, at the receiver front-end to zero the incoming signal samples that exceed a certain threshold [6], [7].
To our knowledge, existing work on this topic concerns only OFDM-based systems [8], [9]. In contrast, in this paper we investigate the application of multi-carrier CDMA (MC-CDMA) with blanking. While both MC-CDMA and conventional OFDM transmit symbols in parallel, they differ in the sense that the latter transmits different symbols over the available sub-carriers whereas in the former each symbol is spread over the entire number of sub-carriers. The motivation for using CDMA for PLC resides in its flexibility as a multiple access and resilience to impulsive noise, as well as its lower peak-to-average power ratio (PAPR) features, compared to OFDM, for certain types of sequences. To realize MC-CDMA, [10], [11], many classes of spreading sequences can be utilized such as pseudonoise (PN), Gold, Kasami, Walsh-Hadamard (WH) and poly-phase (PP) sequences, which have different properties particularly with respect to auto-correlation and cross-correlation functions.

As for this paper, for conciseness, but without loss of generality, we focus on the PN, WH and PP sequences. The rationale for selecting these codes is, firstly, because they are commonly used in CDMA-based systems. Secondly, such codes represent the majority of spreading sequences which are mainly categorized into orthogonal and non-orthogonal codes [12], in addition to their auto-correlation and cross-correlation properties. For example, PN codes [13], have Gaussian-like autocorrelation function, which is an advantage, but exhibit poor cross-correlation between the codes. In contrast, binary WH codes, [14], have zero cross-correlation, in the case of perfect synchronization, making it very attractive for the downlink transmission. However, the resultant large peak power when the number of users is very small and the limited number of available codes constrained by the code length, as well as their sensitivity to time-misalignments, remain the main drawbacks of WH sequences [15], [16]. As for PP sequences, they are the non-binary extension of the binary $\mathrm{WH}$ sequences. As well as having similar cross-correlation properties as the binary $\mathrm{WH}$, their advantages include robustness against timing misalignment which can significantly degrade the performance of the binary WH codes. It is also worth mentioning that, unlike WH sequences, PP codes are not limited by the sequence length and tend to have better PAPR properties. The latter property, particularly, is of great importance since reducing the PAPR 


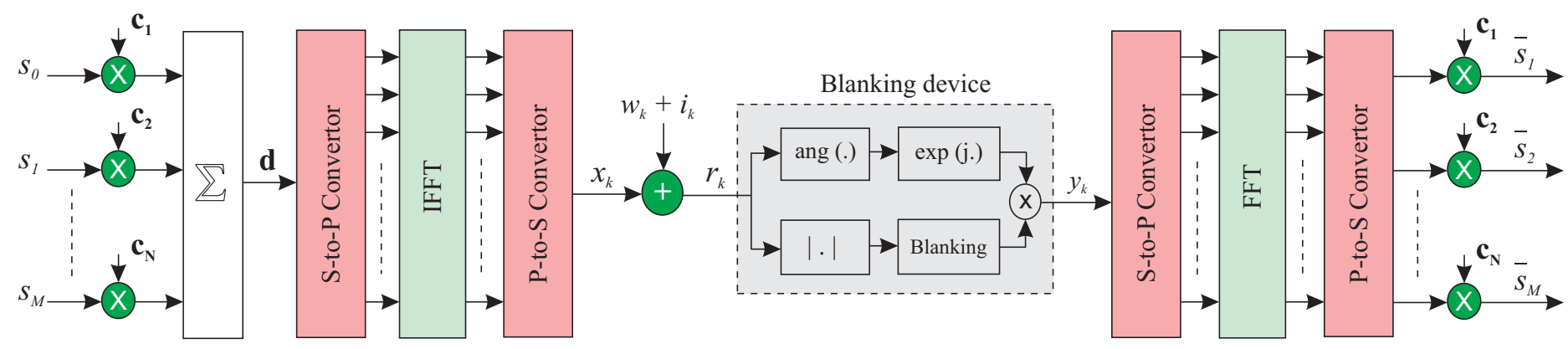

Figure 1: Block diagram of MC-CDMA system with a blanking device at the receiver.

implies that more of the transmitted signal energy is contained close to the average peak value making impulsive noise more distinguishable at the receiver as will be shown later.

Therefore, the contribution of this paper is as follows. First it investigates the PAPR performance of PN, WH, and PP based MC-CDMA systems, then considers the probability of blanking error $\left(P_{b}\right)$ under full-loading (FL) and half-loading (HL) system scenarios, i.e. all or half of the users in the system are active, respectively. The second contribution resides in evaluating the signal-to-noise ratio (SNR) value at the output of the blanking device subject to the various aforementioned loadings. For the sake of completeness and comparison, we have included the performance of the OFDM scheme throughout our investigations. The results show that MC-CDMA in conjunction with blanking at the receiver is able to outperform the OFDM blanking-based system under the FL scenario. Furthermore, it is found that the MC-CDMA-PP system offers the best performance in terms of output SNR relative to the OFDM, MC-CDMA-PN and MCCDMA-WH schemes.

The remainder of this paper is structured as follows. In Section II the system model is presented. In Section III, the complementary cumulative distribution function (CCDF) for the proposed MC-CDMA system is discussed with different spreading codes and various loading scenarios whereas $P_{b}$ is analyzed in Section IV. Section V is dedicated to study the SNR at the output of the blanker as a function of the blanking threshold. Finally, conclusions are drawn in Section VI.

\section{System ModeL}

The general layout of the system under consideration is shown in Fig. 1. First, the information bits for each user are mapped into 16-QAM symbols $s_{m}$, where $m=$ $[1,2, \ldots, M]$ and $M$ is the total number of users. After that each symbol is spread using the user-specific code $\mathbf{c}_{m}=$ $\left[c_{m}^{(0)}, c_{m}^{(1)}, \ldots, c_{m}^{(N-1)}\right]$, where $N$ denotes the code length. It should be highlighted here that, throughout our investigations unless explicitly stated otherwise, we adopt PP codes with four phases $(\phi=4)$. The spread signals are then multiplexed to produce $\mathbf{d}=\left[d_{0}, d_{1}, \ldots, d_{N-1}\right]$ which is then passed through a serial-to-parallel (S-to-P) convertor. After that, this signal is fed to the inverse fast Fourier transform (IFFT), the size of which is equal to the code length $(N)$, and then applied to a parallel- to-serial (P-to-S) convertor before transmission. The transmitted signal for one MC-CDMA block is expressed mathematically as

$$
x(t)=\sum_{i=0}^{N-1} \sum_{m=0}^{M-1} s_{m} c_{m}^{(i)} e^{\left(j 2 \pi \frac{i}{T_{s}} t\right)}
$$

where $T_{s}$ denotes the MC-CDMA symbol duration. In this paper, oversampling of 4 is applied as such oversampling rate is shown in the literature to provide accurate estimates of the PAPR [17]. Therefore, the PAPR of the MC-CDMA transmitted signal can be expressed in decibels as

$$
\text { PAPR }=10 \log _{10}\left(\frac{\max _{k=0,1, \ldots, N L-1}\left|x_{k}\right|^{2}}{\frac{1}{N L} \sum_{k=0}^{N L-1}\left|x_{k}\right|^{2}}\right)
$$

where $L$ is the oversampling factor and $x_{k}$ is the discrete form of $x(t)$. It is worthwhile pointing out the fact that the oversampling process would significantly increase the computational complexity as more processing is performed [18]. As stated earlier, the noise over PL channels is composed of background noise and impulsive noise

$$
n_{k}=w_{k}+i_{k}, \quad k=0,1,2, \ldots, N L-1
$$

while $n_{k}$ is the total noise component, $w_{k}$ is the additive white Gaussian noise (AWGN) and $i_{k}$ is the impulsive noise. Also, $x_{k}, w_{k}$ and $i_{k}$ are assumed to be mutually independent. To characterize the impulsive noise, in this paper we adopt the twomixture Gaussian model [19]

$$
i_{k}=b_{k} g_{k}, \quad k=0,1,2, \ldots, N L-1
$$

$g_{k}$ is complex white Gaussian noise with mean zero and $b_{k}$ is the Bernoulli process with probability $P\left(b_{k}=1\right)=p$, where $p$ is referred to as the impulsive noise probability of occurrence. Therefore, the probability density function (PDF) of the total noise can be expressed as

$$
f\left(n_{k}\right)=(1-p) \mathcal{G}\left(n_{k}, 0, \sigma_{w}^{2}\right)+p \mathcal{G}\left(n_{k}, 0, \sigma_{w}^{2}+\sigma_{i}^{2}\right)
$$




$$
\begin{aligned}
\mathbb{E}\left[A_{n}^{2}\right]=2(1-p)\left[\sigma_{w}^{2}\left(1-\sigma_{w}^{2}\right)(\right. & \left.\frac{T^{2}}{2\left(1+\sigma_{w}^{2}\right)}+1\right) e^{\left.-\frac{T^{2}}{2\left(1+\sigma_{w}^{2}\right)}\right]} \\
& +2 p\left[\left(\sigma_{w}^{2}+\sigma_{i}^{2}\right)+\left(1-\sigma_{w}^{2}-\sigma_{i}^{2}\right)\left(\frac{T^{2}}{2\left(1+\sigma_{w}^{2}+\sigma_{i}^{2}\right)}+1\right) e^{-\frac{T^{2}}{2\left(1+\sigma_{w}^{2}+\sigma_{i}^{2}\right)}}\right]
\end{aligned}
$$

where $\mathcal{G}($.$) is the Gaussian PDF given as \mathcal{G}\left(y, \mu, \sigma_{y}^{2}\right)=$ $\frac{1}{\sqrt{2 \pi \sigma_{y}^{2}}} \exp \left(-\frac{(y-\mu)^{2}}{2 \sigma_{y}^{2}}\right) \cdot \sigma_{w}^{2}$ is the AWGN variance and defines the input SNR as SNR $=10 \log _{10}\left(\frac{\sigma_{x}^{2}}{\sigma_{w}^{2}}\right)$ whereas $\sigma_{i}^{2}$ denotes the impulsive noise variance and defines the signal-to-impulsive noise ratio (SINR) as SINR $=10 \log _{10}\left(\frac{\sigma_{x}^{2}}{\sigma_{i}^{2}}\right)$.

Assuming a perfect synchronization condition, and depending on whether impulsive noise is present or not, the received signal has the following form

$$
r_{k}=\left\{\begin{array}{ll}
x_{k}+w_{k}, & \mathcal{H}_{0} \\
x_{k}+w_{k}+i_{k}, & \mathcal{H}_{1}
\end{array} \quad k=0,1, \ldots, N L-1\right.
$$

The null hypothesis $\mathcal{H}_{0}$ and the alternative hypothesis $\mathcal{H}_{1}$ imply the absence $\left\{P\left(\mathcal{H}_{0}\right)=(1-p)\right\}$ and presence $\left\{P\left(\mathcal{H}_{1}\right)=p\right\}$ of impulsive noise, respectively. At the receiver front-end, blanking is performed as

$$
y_{k}=\left\{\begin{array}{ll}
r_{k}, & \left|r_{k}\right| \leq T \\
0, & \left|r_{k}\right|>T
\end{array} \quad k=0,1, \ldots, N L-1\right.
$$

while $T$ is the blanking threshold, $r_{k}$ and $y_{k}$ are the input and output of the blanker, respectively, as shown in Fig. 1; the operators: ang $($.$) and \exp (j$.$) in this figure are used to extract$ and preserve the phase of the received signal since only the signal amplitude values are processed. It must be noted that determining the blanking threshold value is crucial for achieving best performance since, on one hand, using a too low value for $T$ will result in zeroing most of the useful signal; on the other hand, a very large value for $T$ implies overlooking most of the impulsive noise which can also degrade the system performance significantly. For the blanking-based OFDM systems, Zhidkov derived in [6] a closed-form expression for the output SNR as a function of the impulsive noise parameters, and is given by

$$
\mathrm{SNR}_{\mathrm{OFDM}}=\frac{2}{\mathbb{E}\left[A_{n}^{2}\right]}
$$

where $\mathbb{E}\left[A_{n}^{2}\right]$ is given by (8). This expression will be used later to validate the accuracy of our simulations and also to provide a comparative study with the proposed MC-CDMA based systems. After the noise suppression stage, signal detection takes place as shown in Fig. 1. First, $y_{k}$ is passed through a S-to-P convertor then applied to the fast Fourier transform (FFT). After that, the output of the FFT is multiplied by the spreading codes to produce estimates for the data symbols of the different users $\bar{s}_{m}$.

\section{Complementary Cumulative Distribution FUNCTION (CCDF)}

The CCDF of PAPR is a good measure of the signal peak distribution and is defined as the probability that the PAPR of a data block exceeds a given threshold $\left(\mathrm{PAPR}_{o}\right)$. It is mathematically expressed as

$$
\mathrm{CCDF}=1-\operatorname{Pr}\left(\mathrm{PAPR} \leq \mathrm{PAPR}_{o}\right)
$$

Some simulation results for the CCDF of the MC-CDMA system are illustrated in Fig. 2 with various spreading codes for FL (64 users) and HL (32 users) scenarios. The reason why we consider different loadings is because in practice such scenarios can take place since not all the users are always active, i.e. continuously transmitting. In these simulations, 100,000 random symbols are generated and the corresponding symbol peaks are calculated. All our investigations from this point onward are based on 16-QAM modulation, $N=64$ subcarriers, and the MC-CDMA signal power is normalized as $\sigma_{x}^{2}=(1 / 2) E\left[\left|x_{k}\right|^{2}\right]=1$. For the sake of comparison, results for the OFDM system are also plotted in Fig. 2 and it should be mentioned the analytical results of this system are found using [20]

$$
\mathrm{CCDF}=1-\left(1-e^{\left(-\mathrm{PAPR}_{o}\right)}\right)^{N}
$$

where $N$ is the number of sub-carriers. As apparent from the results in Fig. 2, the analytical results of the OFDM system and the simulated ones are in good agreement. It is also noticeable for FL that MC-CDMA-WH and MC-CDMA-PP schemes have considerably lower PAPR compared to OFDM while the PPbased system offers the best performance. For instance, at $\mathrm{CCDF}=10^{-3}$ MC-CDMA with $\mathrm{WH}$ and $\mathrm{PP}$ codes provide a PAPR reduction of about $1 \mathrm{~dB}$ and $1.5 \mathrm{~dB}$, respectively. In addition, it can be deduced that MC-CDMA-PN exhibits similar performance to OFDM which could be a result of the high cross-correlation property of such codes. Interestingly enough, however, both MC-CDMA-PN and OFDM schemes are invariant to system loading; hence, for succinctness, only the FL scenario for these two systems will be considered throughout the rest of this paper.

Furthermore, it is observed that as the number of PP codes phases is increased the performance is enhanced providing a gain of up to $2.5 \mathrm{~dB}$ when $(\phi=8)$, compared to OFDM, at $\mathrm{CCDF}=10^{-3}$. This can be intuitively justified by the increase in the phase randomization across the sub-carriers which is related to the minimum-distance decoding of the sequences [21]. This, eventually, leads to more averaging of the transmitted MCCDMA signal and, consequently, improves the overall PAPR 


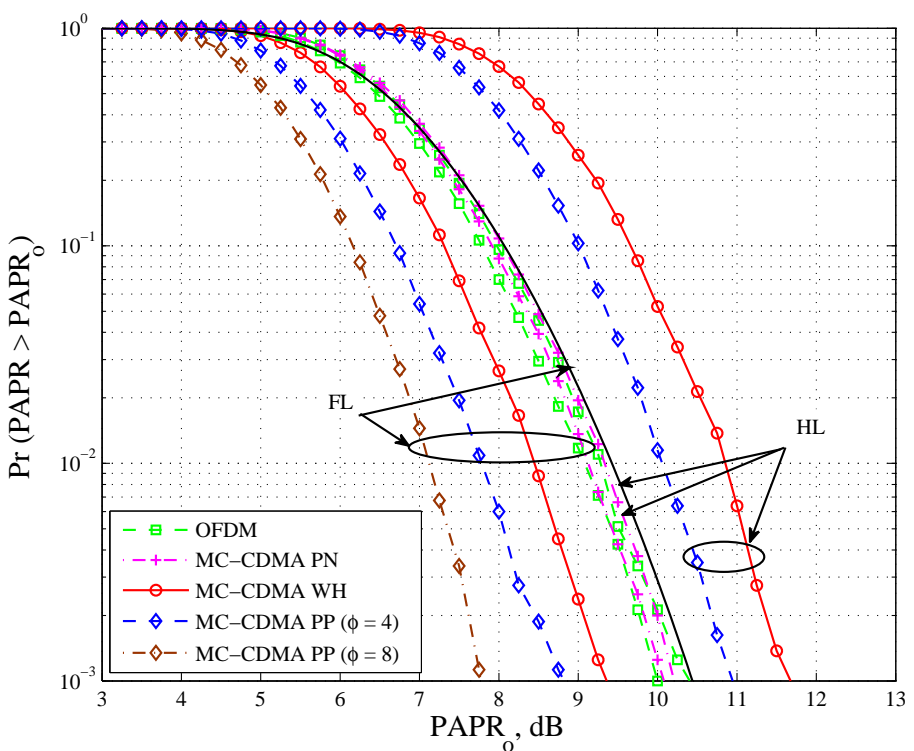

Figure 2: CCDF plot of the PAPR for the MC-CDMA and OFDM systems with FL and HL; (black-solid line represents the analytical results for FL OFDM).

performance. As a result, better probability of blanking error can be achieved, i.e. more efficient noise suppression can be accomplished as shown below.

\section{Probability of Blanking ERror}

$P_{b}$ is defined as the probability that the amplitude of the received sample, $A_{r}=\left|r_{k}\right|$, exceeds $T$ when it is unaffected by impulsive noise and is given by the joint probability $P\left(B, \mathcal{H}_{0}\right)$, where $B$ is the event of blanking the received signal

$$
P_{b}=P\left(B, \mathcal{H}_{0}\right)=\operatorname{Pr}\left(A_{r}>T \mid \mathcal{H}_{0}\right) P\left(\mathcal{H}_{0}\right)
$$

For MC-CDMA with PN, WH and PP sequences, $P_{b}$ is found by means of simulation by satisfying

$$
P_{b}=\int_{T}^{\infty} f_{A_{r}}\left(r \mid \mathcal{H}_{0}\right) \mathrm{dr}
$$

where $f_{A_{r}}\left(r \mid \mathcal{H}_{0}\right)$ is the PDF of the MC-CDMA signal amplitudes in the absence of impulsive noise. Fig. 3 shows $P_{b}$ as a function of $T$ for the MC-CDMA and OFDM approaches with HL and FL. The analytical results of the OFDM system are obtained from (14) since the received OFDM signal, when impulsive noise is absent, has Rayleigh distribution with parameter $\sigma^{2}=\sigma_{s}^{2}+\sigma_{w}^{2}$. It is obvious that these results correlate well with the simulated ones.

$$
P_{b}^{o f d m}=\exp \left(-\frac{T^{2}}{2\left(\sigma_{s}^{2}+\sigma_{w}^{2}\right)}\right)(1-p)
$$

It is clear that for all the systems under consideration, $P_{b}$ improves as $T$ is increased. As anticipated, under FL condition, the WH- and PP-based techniques outperform the OFDM scheme whereas the opposite is true for HL. For instance, at $T=3.5$ MC-CDMA-PP offers a probability reduction of about 1 order

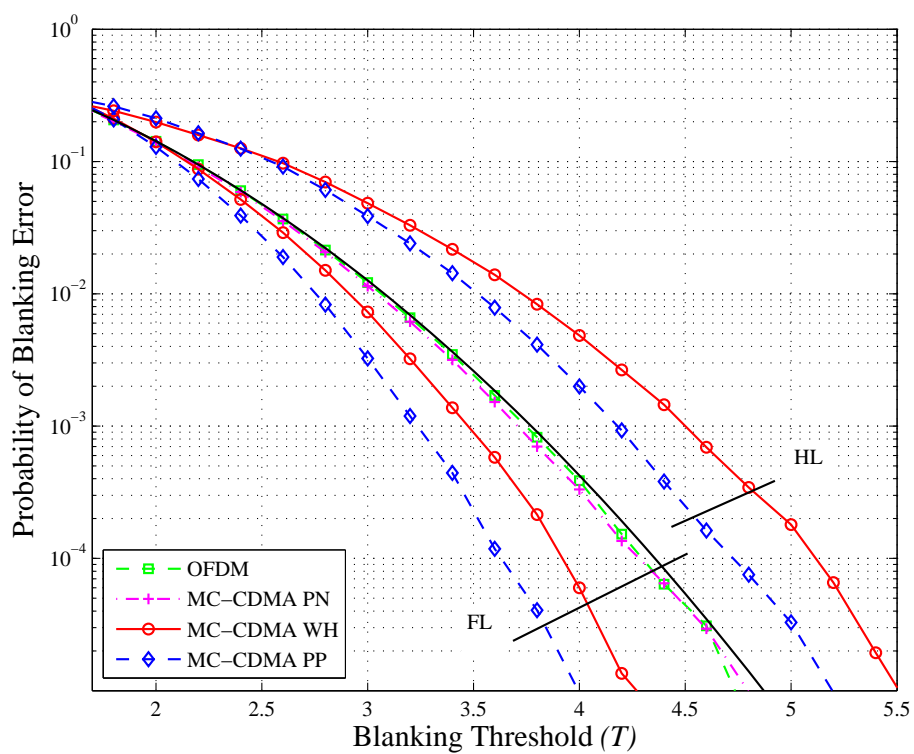

Figure 3: Probability of blanking error versus $T$ for MC-CDMA-PN, MCCDMA-WH, MC-CDMA-PP $(\phi=4)$ and OFDM with FL and HL when input $\mathrm{SNR}=25 \mathrm{~dB}$; (black-solid line represents the analytical results for FL OFDM).

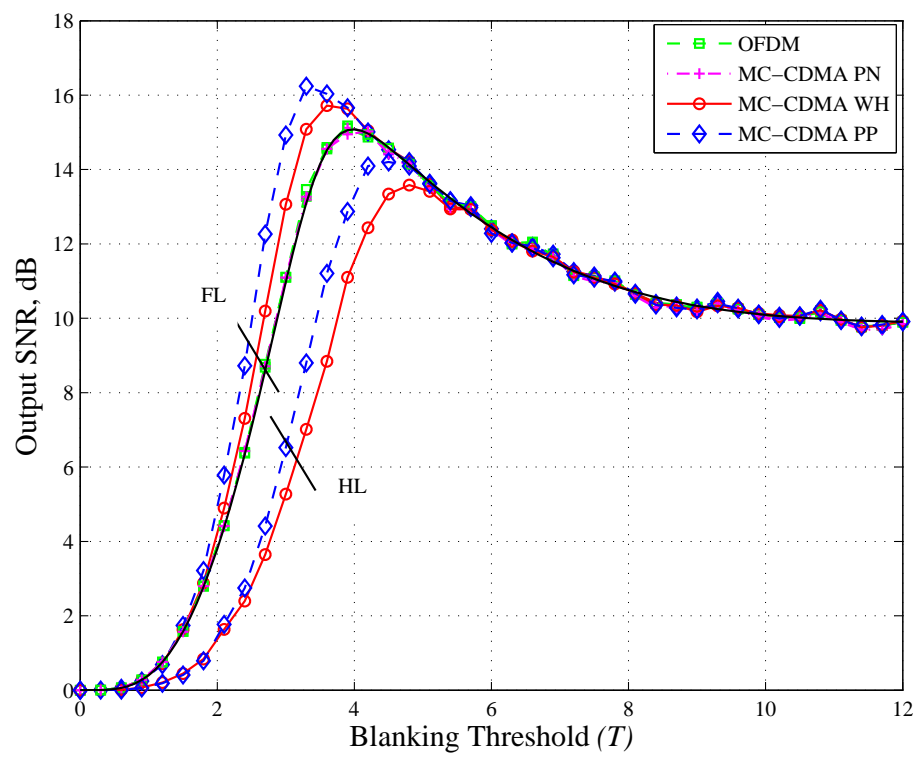

Figure 4: Output SNR versus $T$ for MC-CDMA-PN, MC-CDMA-WH, MC-CDMA-PP $(\phi=4)$ and OFDM with FL and HL when SINR = $-10 \mathrm{~dB}, p=0.01$ and input $\mathrm{SNR}=25 \mathrm{~dB}$; (black-solid line represents the analytical results for FL OFDM (9)).

of magnitude in FL scenario while worsening the performance by about 0.5 order of magnitude for HL compared to OFDM. In addition, it is shown that the MC-CDMA-PN approach has same performance as conventional OFDM. Although the probability of blanking error is, generally, a useful performance measure, the overall system performance is impacted not only by the number of erroneously blanked samples but also by the lost energy contained within these samples. Therefore, it must be emphasized here that more quantitative aspects of the achievable performance should also be investigated such as SNR at the 


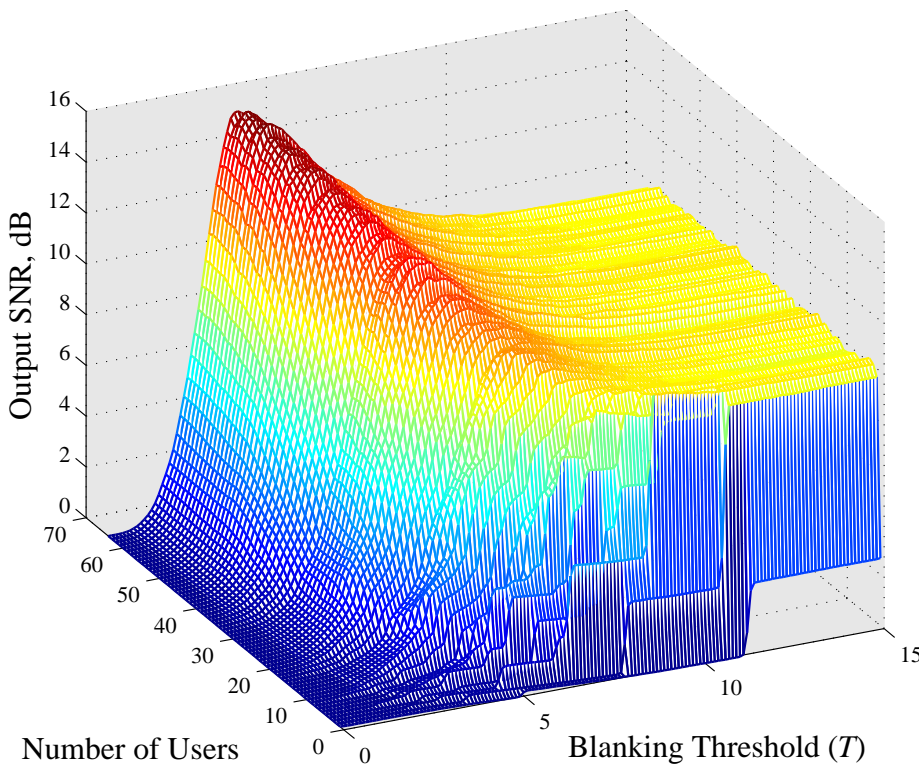

Figure 5: Surface plot of the output SNR as a function of the number of active users and the blanking threshold when SINR $=-10 \mathrm{~dB}, p=0.01$ and input $\mathrm{SNR}=25 \mathrm{~dB}$.

output of the blanking device and/or SER performance.

\section{OUtPut SNR AS A FunCtion OF THE BLANKING THRESHOLD}

In this section, we evaluate the output SNR performance of the proposed system which is determined as

$$
\mathrm{SNR}_{\text {out }}=\frac{\mathbb{E}\left[\left|x_{k}\right|^{2}\right]}{\mathbb{E}\left[\left|y_{k}-x_{k}\right|^{2}\right]}
$$

Fig. 4 depicts the output SNR of the MC-CDMA approach with PN, WH and PP codes for FL and HL as a function of $T$ when input $\mathrm{SNR}=25 \mathrm{~dB}, \mathrm{SINR}=-10 \mathrm{~dB}$ and $p=0.01$. Such values represent a medium-disturbed PLC channel over which the impulsive noise power is around $35 \mathrm{~dB}$ above the background noise level with probability of occurrence of about $1 \%$ [22]. Analytical and simulated results for the OFDM-based system are also included on this plot. It is evident that in a FL scenario the MC-CDMA-PP and MC-CDMA-WH approaches provide SNR gains of up to $1.2 \mathrm{~dB}$ and $0.5 \mathrm{~dB}$ relative to the OFDM-based scheme, respectively. It is also clear that all the systems considered suffer from a dramatic performance degradation when $T$ is too low and similar observations can be seen at the other extreme, i.e. when $T \rightarrow \infty$. For better illustration of the effect of system loading and blanking threshold on the output SNR, we present in Fig. 5 a 3D surface plot for the output SNR of a WH-based system as a function of the number of active users and the blanking threshold. These results clearly indicate that increasing the system load in the MC-CDMA system will make impulsive noise cancellation more efficient, and vice versa.

\section{CONCLUSION}

In this paper we looked into the performance of MC-CDMA approach over PL channels combined with blanking at the receiver to reduce the impact of impulsive noise. Three spreading sequences, namely: PN, WH and PP codes were investigated under various system loading scenarios. It was found that under full-loading condition the proposed system with WH and PP codes always outperforms the conventional OFDM blankingbased scheme in terms of probability of blanking error and output SNR performance for all noise scenario under consideration.

\section{REFERENCES}

[1] E. Del Re, R. Fantacci, S. Morosi, and R. Seravalle, "Comparison of CDMA and OFDM techniques for downstream power-line communications on low voltage grid," IEEE Trans. Power Del., vol. 18, no. 4, pp. $1104-1109$, Oct. 2003

[2] D. Rabphaeli and E. Bassin, "A comparison between OFDM, single carrier and spread for high data rate PLC," in Proc. Int. Symp. Power Lines Commun. (ISPLC), Mar. 1999.

[3] W. Schulz and S. Schwarze, "Comparison of CDMA and OFDM for data communications on the medium voltage power grid," in Proc. Int. Symp. Power Lines Commun. (ISPLC), Apr. 2000.

[4] Y. H. Ma, P. So, and E. Gunawan, "Comparison of CDMA and OFDM systems for broadband power line communications," IEEE Trans. Power Del., vol. 23, no. 4, pp. 1876-1885, Oct. 2008.

[5] M. Hazen, "The technology behind HomePlug AV powerline communications," Computer, vol. 41, no. 6, pp. 90-92, Jun. 2008.

[6] S. V. Zhidkov, "On the analysis of OFDM receiver with blanking nonlinearity in impulsive noise channels," in Proc. Int. Symp. Intell. Signal Process. Commun. Syst., Nov. 2004, pp. 492-496.

[7] E. Alsusa and K. Rabie, "Dynamic peak-based threshold estimation method for mitigating impulsive noise in power-line communication systems," IEEE Trans. Power Del., vol. 28, no. 4, pp. 2201-2208, Oct. 2013.

[8] K. Rabie and E. Alsusa, "Preprocessing-based impulsive noise reduction for power-line communications," IEEE Trans. Power Del., vol. 29, no. 4, pp. 1648-1658, Aug. 2014.

[9] S. V. Zhidkov, "Analysis and comparison of several simple impulsive noise mitigation schemes for OFDM receivers," IEEE Trans. Commun., vol. 56, no. 1, pp. 5-9, Jan. 2008.

[10] S. Hara and R. Prasad, "Overview of multicarrier CDMA," IEEE Commun. Mag., vol. 35, no. 12, pp. 126-133, Dec. 1997.

[11] P. Katsis, G. Papadopoulos, and F.-N. PavJidou, "Coded MC-CDMA systems for power line communications," in Proc. IEEE Int. Conf. on Telecommun. in Modern Satellite, Cable and Broadcast. Service, vol. 1, Oct. 2003, pp. 153-156.

[12] A. Goldsmith, Wireless Communications. 1st ed. Cambridge University Press, 2005

[13] R. Kirlin, "Pseudo-random analog noise generation," Proc. IEEE, vol. 64, no. 5, pp. 824-826, May 1976.

[14] A. Viterbi, "Very low rate convolution codes for maximum theoretical performance of spread-spectrum multiple-access channels," IEEE J. Sel. Areas Commun., vol. 8, no. 4, pp. 641-649, May 1990.

[15] L. Hanzo and T. Keller, "OFDM and MC-CDMA," John Wiley \& Sons, 2006.

[16] L. Yang and E. Alsusa, "Dynamic code-allocation based PAPR reduction technique for MC-CDMA systems," in Proc. IEEE Wireless Commun. Netw. Conf. (WCNC), Mar. 2007, pp. 627-632.

[17] J. Tellado, Multicarrier Modulation With Low PAR: Applications to DSL and Wireless. Norwell, MA: Kluwer, 2000.

[18] C.-L. Wang, S.-J. Ku, and C.-J. Yang, "A low-complexity PAPR estimation scheme for OFDM signals and its application to SLM-based PAPR reduction," IEEE J. Sel. Topics Signal Process., vol. 4, no. 3, pp. 637645, Apr. 2010.

[19] M. Ghosh, "Analysis of the effect of impulse noise on multicarrier and single carrier QAM systems," IEEE Trans. Commun., vol. 44, no. 2, pp. 145-147, Feb. 1996.

[20] R. van Nee and R. Prasad, "OFDM for wireless multimedia communications," Artech House, 2000. 
[21] V. Tarokh and H. Jafarkhani, "On the computation and reduction of the peak-to-average power ratio in multicarrier communications," IEEE Trans. Commun., vol. 48, no. 1, pp. 37-44, Jan. 2000.
[22] M. Zimmermann and K. Dostert, "Analysis and modeling of impulsive noise in broad-band powerline communications," IEEE Trans. Electromagn. Compat., vol. 44, no. 1, pp. 249-258, Feb. 2002. 